\title{
Small intestinal bacterial overgrowth in Alzheimer's disease
}

\author{
Karol Kowalski ${ }^{1} \cdot$ Agata Mulak $^{1}$ (1)
}

Received: 23 April 2021 / Accepted: 4 November 2021 / Published online: 19 November 2021

(c) The Author(s) 2021

\begin{abstract}
The results of animal studies and clinical data support the gut microbiota contribution to the pathogenesis of Alzheimer's disease (AD). The aim of this pilot study was to evaluate the prevalence of small intestinal bacterial overgrowth (SIBO) and fecal markers of intestinal inflammation and permeability in AD patients. The study was conducted in $45 \mathrm{AD}$ patients and 27 controls. Data on comorbidities, pharmacotherapy, and gastrointestinal symptoms were acquired from medical records and a questionnaire. SIBO was evaluated using lactulose hydrogen breath test. Fecal calprotectin and zonulin levels were assessed by ELISA assays. The positive result of SIBO breath test was found in $49 \%$ of the AD patients and $22 \%$ of the controls $(p=0.025)$. The comparative analysis between SIBO-positive and SIBO-negative AD patients with respect to the degree of cognitive impairment, comorbidities and used medications did not reveal any statistically significant difference, except for less common heartburn in SIBO-positive AD patients than in SIBO-negative ones (9 vs 35\%, $p=0.038$ ). The median fecal calprotectin and zonulin levels in the $\mathrm{AD}$ group compared to the control group amounted to $43.1 \mathrm{vs} 64.2 \mu \mathrm{g} / \mathrm{g}(p=0.846)$ and $73.5 \mathrm{vs} 49.0 \mathrm{ng} / \mathrm{ml}(p=0.177)$, respectively. In the AD patients there was no association between the presence of SIBO and fecal calprotectin level. Patients with AD are characterized by higher prevalence of SIBO not associated with increased fecal calprotectin level that may be related to anti-inflammatory effect of cholinergic drugs used in the treatment of AD.
\end{abstract}

Keywords Alzheimer's disease $\cdot$ Brain-gut-microbiota axis $\cdot$ Calprotectin $\cdot$ Small intestinal bacterial overgrowth

\section{Introduction}

Alzheimer's disease (AD) is the most common cause of dementia characterized by a progressive decline in cognitive function (Reitz and Mayeux 2014). The main features of the disease are aggregation, oligomerization, and deposition of amyloid beta $(A \beta)$ in the form of plaques as well as formation of neurofibrillary tangles composed of hyperphosphorylated tau protein (Jouanne et al. 2017). Those deposits induce neuroinflammation leading to the synapse loss and neuronal cell death (Köhler et al. 2016). Despite large amount of data on the $\mathrm{A} \beta$ role in $\mathrm{AD}$ it is still not well known what triggers the amyloid aggregation and plaque formation. Besides the complex biochemical processes involved in the neuronal degeneration, other molecular and neurochemical alterations occur including cholinergic deficit due to basal forebrain degeneration (Martorana et al. 2010).

Agata Mulak

agata.mulak@wp.pl

1 Department of Gastroenterology and Hepatology, Wroclaw Medical University, Borowska 213, 50-556 Wrocław, Poland
Since acetylcholine is involved in cognitive processes, the so-called cholinergic hypothesis has been proposed according to which an increase in acetylcholine level could restore cognitive deficits (Hampel et al. 2018). In fact, most currently available drug therapies, like acetylcholinesterase inhibitors, are still based on this hypothesis (Ferreira-Vieira et al. 2016). However, the current symptomatic treatment does not cure the disease, therefore, there is a need for new therapeutic strategies targeted at the mechanisms involved in the pathogenesis of AD (Zhu et al.2020).

The gut microbiota is a key element in bidirectional interactions between the central nervous system (CNS) and the gastrointestinal tract (Köhler et al. 2016; Dinan and Cryan 2017; Quigley 2017). Recently, disturbances within the brain-gut-microbiota axis, including the CNS and the enteric nervous system (ENS), have been shown to contribute to the pathogenesis of $\mathrm{AD}$ (Kowalski and Mulak 2019). The formation of $A \beta$ takes place both in the CNS and the ENS (Puig and Combs 2013; Semar et al. 2013). In addition, a large amount of amyloids is produced by the gut microbiota (Zhao et al. 2015). Bacterial amyloids differ from the CNS amyloids in their primary structure but share 
similarities in their tertiary structure (Friedland and Chapman 2017). Through molecular mimicry bacterial amyloids may act as prion proteins that induce misfolding of native amyloids leading to their aggregation and deposition in amyloid plaques (Friedland 2015).

The gut microbiota is known to upregulate local and systemic inflammation due to lipopolysaccharides (LPS) from pathogenic bacteria and synthesis of pro-inflammatory cytokines (Lukiw 2016; Zhao et al. 2017; Zhan et al. 2018). The gut dysbiosis may induce increased permeability of the intestinal barrier and the blood-brain barrier further enhancing inflammation at the gut, systemic, and CNS levels (Köhler et al. 2016; Kowalski and Mulak 2019). Alterations in the gut microbiota composition characterized by its decreased diversity and stability contribute to overstimulation of immune system and induction of chronic inflammation observed in the elderly (Jouanne et al. 2017). Age-dependent blood-brain barrier changes, especially the increased permeability in the hippocampus involved in learning and memory processes, allow blood-derived molecules to enter the brain (Zhao et al. 2017). The bacterial overgrowth in the small intestine, where permeability is physiologically higher, might be a source of bacterial products, including amyloids and LPS.

The results of studies in animal models and clinical data support the gut microbiota contribution to the pathogenesis of AD (Köhler et al. 2016; Quigley 2017; Kowalski and Mulak 2019; He et al. 2020; Liu et al. 2020; Sochocka et al. 2019). To the best of our knowledge, until now no data on quantitative microbiota disturbances in $\mathrm{AD}$ have been published, although there are some reports in other neurodegenerative diseases such as Parkinson's disease (Fasano et al. 2013). Similarly, data on intestinal inflammation and permeability markers in $\mathrm{AD}$ are limited (Leblhuber et al. 2015, 2017). Results of these studies may allow to determine new therapeutic strategies in AD which encompass influencing intestinal immune system and gut permeability by qualitative and quantitative modifications of microbial composition.

The aim of this study was to evaluate the prevalence of quantitative microbiota disturbances in the form of small intestinal bacterial overgrowth (SIBO) in AD patients. Additionally, markers of intestinal inflammation and permeability, calprotectin, and zonulin levels, respectively, were assessed in stool samples. The analysis of patients' subgroups with positive and negative breath test results was aimed at evaluating potential relationship between the tested parameters and clinical data including the degree of dementia.

\section{Materials and methods}

\section{Subjects}

The study was conducted in 45 patients $(9 \mathrm{M}, 36 \mathrm{~F})$ with mild or moderate dementia in the course of AD. All patients were recruited among the subjects hospitalized at the single Alzheimer's Disease Research Center. The degree of cognitive impairment was assessed based on the Mini-Mental State Examination (MMSE). The control group consisted of 27 participants ( $8 \mathrm{M}, 19 \mathrm{~F}$ ) matched according to age, sex, and body mass index (BMI) (subjects diagnosed with dementia, other mental disorders and CNS diseases were excluded). Data on comorbidities, pharmacotherapy, and gastrointestinal symptoms were acquired from medical records and a questionnaire. The following features were considered as the exclusion criteria: significant comorbidities regarding the CNS disorders and gastrointestinal diseases, previous gastrointestinal surgery except for appendectomy and cholecystectomy, the use of antibiotics or preparation for colonoscopy within the last month prior the stool collection, and the use of nonsteroidal anti-inflammatory drugs except for cardioprotective low-dose acetylsalicylic acid (ASA) (75 mg daily). All subjects were tested for SIBO with hydrogen breath test and were asked to provide a stool sample. The protocol of this study was approved by the local Ethics Committee (KB-491/2017). A written informed consent was obtained from all participants prior to the study enrollment.

\section{Evaluation of small intestinal bacterial overgrowth (SIBO)}

The presence of SIBO was investigated by the use of lactulose hydrogen breath test (LHBT). The concept of breath testing is based on the exhalation of gases (hydrogen) produced solely by the intestinal bacteria following ingestion of a carbohydrate substrate (Bohm et al. 2013). All study participants underwent LHBT after preparation including diet restriction and fasting period of at least $12 \mathrm{~h}$. The test was performed in the fasted state in the morning, after tooth brushing. Food intake, physical exercise, and smoking were not permitted during testing. Exhaled air samples were analyzed using Gastrolyzer ${ }^{\circledR}$ (Bedfont Scientific Ltd, Kent, UK). The air samples were collected at the baseline and then after oral administration of lactulose (10 $\mathrm{g}$ in $200 \mathrm{~mL}$ of water) at intervals of $15 \mathrm{~min}$ during the period of $90 \mathrm{~min}$. An increase above 20 parts per million (ppm) of expired hydrogen after 90 min compared to the basal value indicated a positive result. 


\section{Quantitative evaluation of fecal biomarkers}

Stool samples of app. $5 \mathrm{~g}$ each were collected according to the instruction. After required preparation the samples were stored at $-20{ }^{\circ} \mathrm{C}$ until processing as described previously (Ohlsson et al. 2017). The quantitative evaluations of fecal calprotectin and zonulin were performed by ELISA tests: EK-CAL (Bühlmann Laboratories, Switzerland) and IDK $^{\circledR}$ Zonulin (Immundiagnostik AG, Germany), respectively. Noteworthy, very recently, during the current study duration, the accuracy of the used zonulin ELISA assay has been questioned (Ajamian et al. 2019) that is adequately addressed in the discussion.

\section{Statistical analysis}

Due to non-normal distribution of the data, non-parametric statistics were used and results are expressed as median along with the lower and upper quartiles (25Q-75Q). The Mann-Whitney $U$ test and chi-squared test were applied to compare differences between the groups. The Spearman's rank correlation coefficient $(R)$ was also calculated to test associations between variables. The value of $p<0.05$ was considered to be statistically significant.

\section{Results}

\section{Subjects' characteristics}

In the $\mathrm{AD}$ patients the median age was 74 years (57-88), median BMI amounted to $26.9 \mathrm{~kg} / \mathrm{m}^{2}$, and women constitute $80 \%$ of the group. There were no statistically significant differences between the above characteristics compared to the control subjects (Table 1). The median MMSE score in the AD patients amounted to 17.0 (14.5-21.5). The most common comorbidities observed in the AD patients as well as in the controls included arterial hypertension (64 vs 63\%, $p=0.899$ ), ischemic heart disease (29 vs $7 \%, p=0.029$ ), hyperlipidemia ( 29 vs $7 \%, p=0.029$ ), and type 2 diabetes (24 vs $33 \%, p=0.414$ ). Mental disorders were present only in the $\mathrm{AD}$ group. Among the drugs used only by the $\mathrm{AD}$ patients were acetylcholinesterase inhibitors, memantine, quetiapine and others neuroleptics, anticonvulsants, and antidepressants. Moreover, the $\mathrm{AD}$ patients more frequently used ASA in the cardioprotective dose of $75 \mathrm{mg}$ daily compared to the controls $(p=0.002)$. There were no other significant differences between the groups regarding pharmacotherapy. The prevalence of gastrointestinal symptoms reported by the $\mathrm{AD}$ patients and the controls is presented in Table 2. Constipation (stool consistency described as type 1 or 2 according to the Bristol Stool Form Scale), although common in both groups, was less prevalent in the AD patients (31 vs $62 \%, p=0.012$ ). Moreover, the AD patients compared to the control subjects less frequently reported heartburn (22 vs $46 \%, p=0.036$ ). However, there was no significant difference between the groups with respect to proton pump inhibitor (PPI) use (16 vs 19\%, $p=0.744$ ).

\section{Breath test results}

The positive result of SIBO breath test was found in $49 \%$ of the $\mathrm{AD}$ patients and $22 \%$ of the control subjects $(p=0.025)$ (Fig. 1). The percentage of women in SIBO-positive and SIBO-negative AD patients was similar (86 vs $74 \%$, $p=0.297)$. Comparing SIBO-positive AD patients $(n=22)$ to SIBO-negative AD patients $(n=23)$ no significant differences in age, BMI, and the MMSE score between these subgroups were found (Table 1). The comparative analysis between those subgroups with respect to comorbidities and used medications did not reveal any statistically significant difference, except for less common heartburn in

Table 1 Basic characteristics of the recruited AD patients and the controls

\begin{tabular}{|c|c|c|c|c|c|c|}
\hline & $\begin{array}{l}\text { AD patients } \\
n=45\end{array}$ & $\begin{array}{l}\text { Controls } \\
n=27\end{array}$ & $p$ & $\begin{array}{l}\mathrm{SIBO}+ \\
\mathrm{AD} \text { patients } \\
n=22\end{array}$ & $\begin{array}{l}\text { SIBO- } \\
\text { AD patients } \\
n=23\end{array}$ & $p$ \\
\hline $\begin{array}{l}\text { Age range (years) } \\
M(25 Q-75 Q)\end{array}$ & $\begin{array}{l}57-88 \\
74.0(68.0-80.0)\end{array}$ & $\begin{array}{l}60-93 \\
72.0(68-76)\end{array}$ & $0.314^{\mathrm{a}}$ & $\begin{array}{l}58-88 \\
75.0(67.8-75.0)\end{array}$ & $\begin{array}{l}57-85 \\
73.0(68.0-78.0)\end{array}$ & $0.313^{\mathrm{a}}$ \\
\hline Males/females & $9 / 36$ & $8 / 19$ & $0.352^{\mathrm{b}}$ & $3 / 19$ & $6 / 17$ & $0.297^{\mathrm{b}}$ \\
\hline $\begin{array}{l}\text { BMI }\left(\mathrm{kg} / \mathrm{m}^{2}\right) \\
\text { M }(25 \mathrm{Q}-75 \mathrm{Q})\end{array}$ & $26.9(23.9-29.8)$ & $27.8(24.6-30.4)$ & $0.412^{\mathrm{a}}$ & $27.0(24.8-29.8)$ & $26.9(23.7-29.6)$ & $0.733^{\mathrm{a}}$ \\
\hline $\begin{array}{l}\text { MMSE (points) } \\
\text { M (25Q-75Q) }\end{array}$ & $17.0(14.5-21.5)$ & - & - & $16.0(14.0-20.0)$ & $17.0(14.5-21.5)$ & $0.723^{\mathrm{a}}$ \\
\hline
\end{tabular}

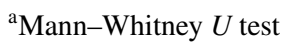

${ }^{\mathrm{b}} \chi^{2}$ test

$B M I$ body mass index, $M$ median, $25 Q-75 Q$ lower and upper quartiles, $M M S E$ Mini-Mental State Examination, SIBO small intestinal bacterial overgrowth 
Table 2 The most common gastrointestinal symptoms reported by the $\mathrm{AD}$ patients $(n=45)$, the controls $(n=26)$, SIBO-positive AD patients $(n=22)$, and SIBO-negative AD patients $(n=23)$

\begin{tabular}{lllllll}
\hline Gastrointestinal symptoms & $\begin{array}{l}\text { AD patients } \\
n(\%)\end{array}$ & $\begin{array}{l}\text { Controls } \\
n(\%)\end{array}$ & $p$ & $\begin{array}{l}\text { SIBO+ } \\
\text { AD patients } \\
n(\%)\end{array}$ & $\begin{array}{l}\text { SIBO- } \\
\text { AD patients } \\
n(\%)\end{array}$ & $p$ \\
\hline Constipation & $14(31)$ & $16(62)$ & $\mathbf{0 . 0 1 2}$ & $6(27)$ & $10(43)$ & 0.605 \\
Diarrhea & $1(2)$ & 0 & 0.444 & 0 & $1(4)$ & 0.915 \\
Disturbed defecation & $14(31)$ & $13(50)$ & 0.114 & $6(27)$ & $8(35)$ & 0.586 \\
Abdominal pain & $10(22)$ & $8(31)$ & 0.425 & $5(23)$ & $5(22)$ & 0.936 \\
Abdominal bloating & $10(22)$ & $8(31)$ & 0.425 & $4(18)$ & $6(26)$ & 0.524 \\
Heartburn & $10(22)$ & $12(46)$ & $\mathbf{0 . 0 3 6}$ & $2(9)$ & $8(35)$ & $\mathbf{0 . 0 3 8}$ \\
\hline
\end{tabular}

$\chi^{2}$ test, $p<0.05$ marked in bold
Fig. 1 Lactulose hydrogen breath test results in the $\mathrm{AD}$ patients and the controls. The prevalence of SIBO was significantly higher in the $\mathrm{AD}$ patients than in age-matched controls without dementia (49 vs $22 \%$, $p=0.025)$. The $p$ value was calculated by the $\chi^{2}$ test. $A D$ Alzheimer's disease

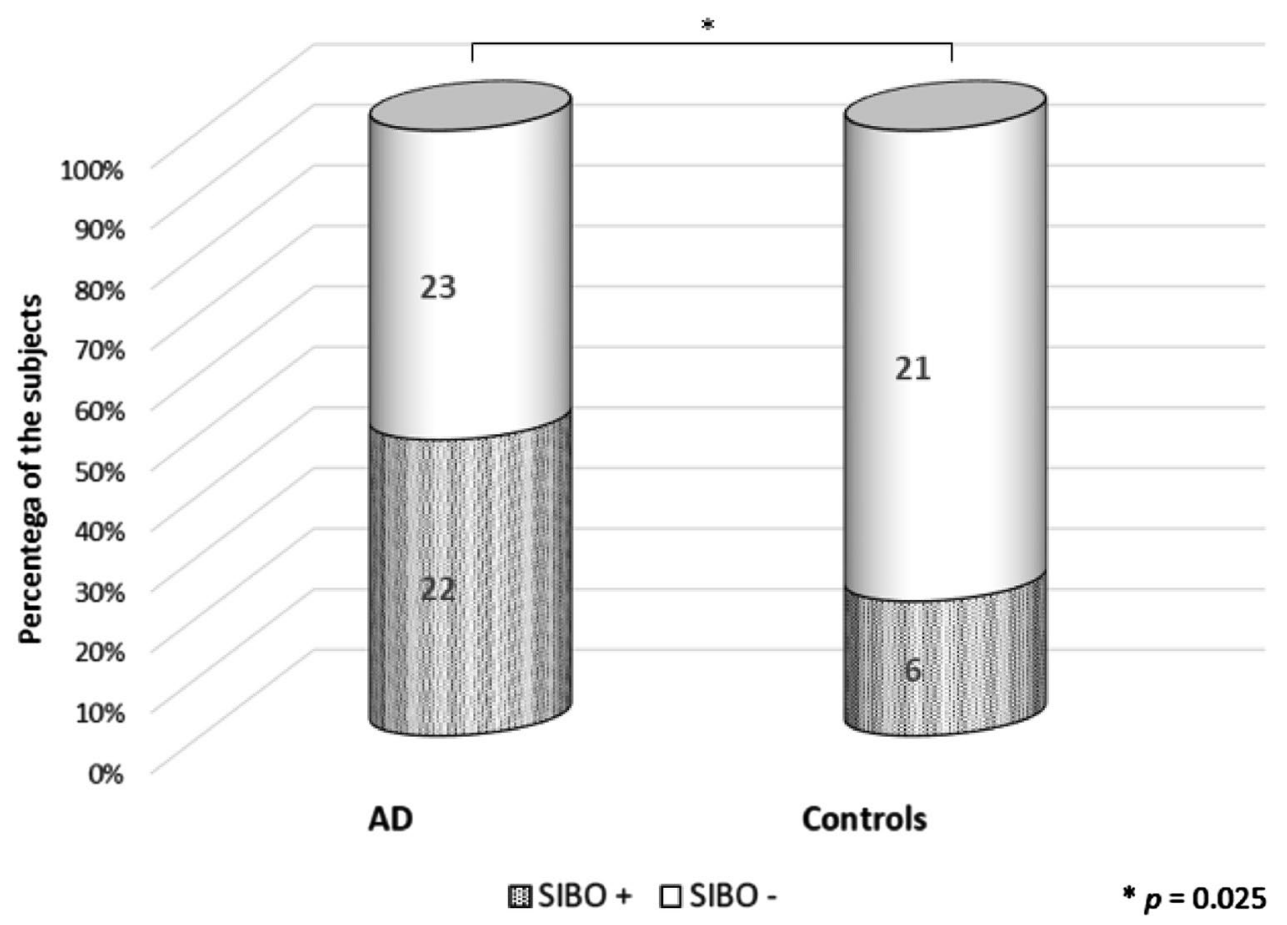

SIBO-positive AD patients than in SIBO-negative ones (9 vs $35 \%, p=0.038$ ). Again, these subgroups did not differ with respect to PPI use (14 vs $17 \%, p=0.728$ ).

\section{Fecal biomarkers}

The stool samples for quantitative evaluations of fecal calprotectin and zonulin were obtained from $35 \mathrm{AD}$ patients and 25 controls. Calprotectin was evaluated in all control subjects, while zonulin only in 16 of them due to aforementioned controversy regarding the accuracy of the zonulin ELISA assay. The median fecal calprotectin and zonulin levels in the AD group compared to the control group amounted to $43.1 \mathrm{vs} 64.2 \mu \mathrm{g} / \mathrm{g}(p=0.846)$ and 73.5 vs $49.0 \mathrm{ng} / \mathrm{ml}(p=0.177)$, respectively (Fig. 2$)$. The normal fecal calprotectin level $<50 \mu \mathrm{g} / \mathrm{g}$ was found in $19 \mathrm{AD}$ patients (54\%) and 10 control subjects (40\%). The numbers of subjects in the above groups with calprotectin levels exceeding $50 \mu \mathrm{g} / \mathrm{g}, 112 \mu \mathrm{g} / \mathrm{g}$ and $150 \mu \mathrm{g} / \mathrm{g}$ are presented in Table 3.

Comparing SIBO-positive AD patients to SIBO-negative AD patients, the median calprotectin and zonulin levels amounted to $52.6 \mathrm{vs} 42.2 \mu \mathrm{g} / \mathrm{g}(p=0.590)$ and $63.0 \mathrm{vs}$ $83.8 \mathrm{ng} / \mathrm{ml}(p=0.568)$, respectively. There was no correlation between the MMSE score and the level of calprotectin $(R=0.22, p=0.306)$ and zonulin $(R=0.05, p=0.808)$. There was no association between calprotectin and zonulin levels and age, BMI, medication use, and gastrointestinal symptom frequency. Among the AD patients, the calprotectin level was significantly higher in those diagnosed with ischemic heart disease $(p=0.017)$, but no other differences regarding comorbidities were found between the subgroups. 


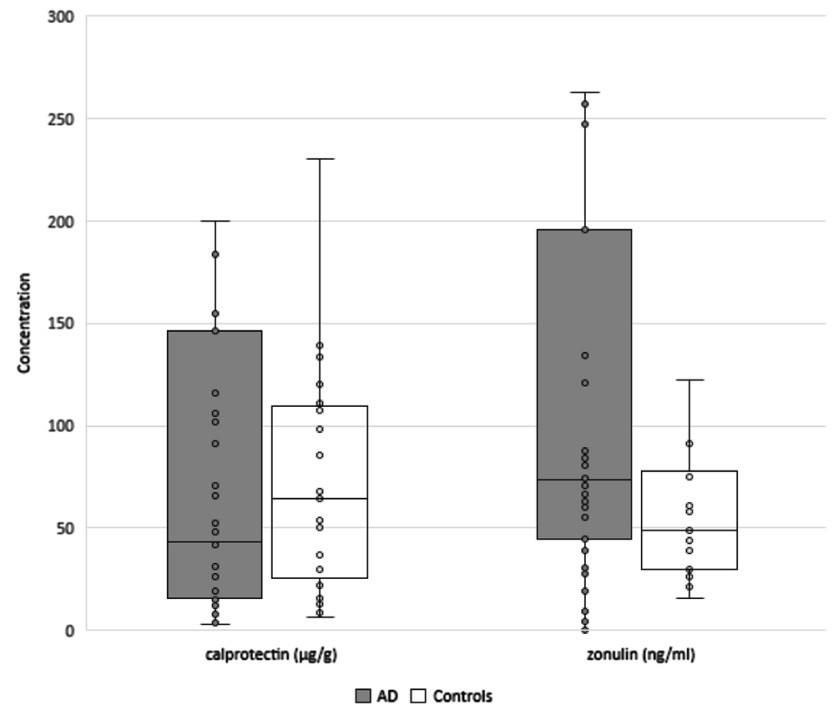

Fig. 2 Comparison of fecal calprotectin and zonulin levels in the AD patients and the controls. The median fecal calprotectin and zonulin levels in the $\mathrm{AD}$ group compared to the control group amounted to $43.1 \mathrm{vs} 64.2 \mu \mathrm{g} / \mathrm{g}(p=0.846)$ and $73.5 \mathrm{vs} 49.0 \mathrm{ng} / \mathrm{ml}(p=0.177)$, respectively. Individual data are presented excluding outliers. The $p$ value was calculated by the Mann-Whitney $U$ test. $\mathrm{AD}$, Alzheimer's disease

Table 3 The comparison of fecal calprotectin levels in the AD patients $(n=35)$ and the controls $(n=25)$

\begin{tabular}{llll}
\hline $\begin{array}{l}\text { Calprotectin } \\
(\mu \mathrm{g} / \mathrm{g})\end{array}$ & $\begin{array}{l}\text { AD patients } \\
n(\%)\end{array}$ & $\begin{array}{l}\text { Controls } \\
n(\%)\end{array}$ & $p$ \\
\hline$<50$ & $19(54)$ & $10(40)$ & 0.275 \\
$\geq 50$ & $16(46)$ & $15(60)$ & \\
$<112$ & $26(74)$ & $20(80)$ & 0.606 \\
$\geq 112$ & $9(26)$ & $5(20)$ & \\
$<150$ & $27(77)$ & $23(92)$ & 0.128 \\
$\geq 150$ & $8(23)$ & $2(8)$ & \\
\hline$\chi^{2}$ test & & &
\end{tabular}

\section{Discussion}

Given the growing understanding of the role of gut microbiota alterations, the gut immune system activation and increased intestinal permeability in $\mathrm{AD}$, the main aim of the study was to evaluate the prevalence of SIBO in the course of $\mathrm{AD}$ and its potential association with fecal calprotectin and zonulin levels. The results of this pilot study showed more common prevalence of SIBO in AD patients compared to the matched controls ( 49 vs $22 \%, p=0.025$ ). No statistically significant differences were found comparing fecal biomarker levels between the AD patients and the controls as well as between the AD patients with positive and negative LHBT results. Although recently numerous studies have been published on the alterations in the gut microbiota composition in AD (Kowalski and Mulak 2019; He et al. 2020; Liu et al. 2020; Sochocka et al. 2019), to the best of our knowledge, this is the first report on SIBO in AD patients, not including our own preliminary study presented in the abstract form (Kowalski et al. 2017).

Regarding the characteristics of the subjects, $80 \%$ of the studied AD patients were women which is consistent with the female predominance in AD (Reitz and Mayeux 2014; Oveisgharan et al. 2018). That may be related to longer life expectancy in women, since age is the main risk factor for dementia (Tom et al. 2015). The most common comorbidities observed in the AD patients included cardiovascular diseases such as arterial hypertension, ischemic heart disease, hyperlipidemia, and type 2 diabetes. A large number of the AD patients were overweight $(45 \%)$ or obese $(20 \%)$. All of the above conditions constitute the risk factors for AD (Armstrong 2019). The control subjects, although less numerous, were matched for sex, age, and BMI. Among concomitant diseases ischemic heart disease and hyperlipidemia were more common in the AD patients, and those factors were linked with $\mathrm{AD}$ in previous research (Armstrong 2019). Moreover, side effects of the medications used in AD include gastrointestinal symptoms. Cholinergic drugs may induce nausea, vomiting, diarrhea or abdominal pain (Colovic et al. 2013), while the use of memantine may result in constipation (Mimica and Presecki 2009). In the studied groups, the significant differences between the AD patients and the controls concerning the frequency of reported gastrointestinal symptoms were less common constipation and heartburn in the AD patients that could potentially be related to a prokinetic effect of cholinergic drugs. As mentioned above there was no difference between the groups regarding the use of PPIs. The fact that the AD patients more frequently than the controls used cardioprotective dose of ASA could be associated with concomitant ischemic heart disease ( 29 vs $7 \%, p=0.029$ ). Nonsteroidal anti-inflammatory drugs can induce an increase in fecal calprotectin level; however, the results of studies regarding this effect with respect to low ASA doses are not consistent (Montalto et al. 2013).

SIBO, assessed by LHBT, was prevalent almost in half of the studied AD patients (49\%) compared to $22 \%$ of the controls $(p=0.025)$. The available data on the SIBO prevalence in the general population or in the course of different disorders are not fully consistent (Quigley 2019). Moreover, some methodological differences may also affect the results. For comparison, in patients with Parkinson's disease (PD) SIBO was reported in 25.3\% (Tan et al. 2014), 30.2\% (Niu et al. 2016), 54.2\% (Gabrielli et al. 2011), and 54.5\% (Fasano et al. 2013) of the subjects. At the same time in the controls included in the above studies SIBO was found in 9.5\% (Niu et al. 2016), 8.3\% (Gabrielli et al. 2011), and 
20.0\% (Fasano et al. 2013). The relatively high percentage of SIBO-positive controls in the current study (22\%) may be associated with more advanced age of the subjects. In general, the majority of studies indicate the age-related increase in the SIBO prevalence (Elphick et al. 2005; Parlesak et al. 2003; Choung et al. 2011), although some of them confirm such a relationship only in women (Newberry et al. 2016). Despite the fact that some conditions like type 2 diabetes or chronic PPI use are well-known risk factors for the development of SIBO, we did not observe such a relation in the current study, probably due to limited number of the subjects. The typical symptoms of SIBO include bloating, flatulence, abdominal pain, diarrhea or constipation. Nevertheless, SIBO is a heterogenous syndrome which may stay asymptomatic in otherwise healthy subjects (Bures et al. 2010; Almeida et al. 2008). In the present study, SIBO in $\mathrm{AD}$ patients was not associated with a higher prevalence of bowel symptoms, similarly to the earlier research conducted in PD patients (Tan et al. 2014). Interestingly, SIBOpositive AD patients were characterized by less common heartburn compared to SIBO-negative AD patients (9 vs $35 \%, p=0.038$ ). Simultaneously, the use of PPI in the both subgroups was comparable (14 vs $17 \%, p=0.728$ ). Presumably, relatively smaller gastric acid secretion protects from heartburn, but predisposes to SIBO. Moreover, in the studied $\mathrm{AD}$ patients, no association between the presence of SIBO and the MMSE score was observed. Interestingly, probiotic supplementation in AD patients have been demonstrated to improve cognitive function assessed by the MMSE (Akbari et al. 2016; Tamtaji et al. 2019).

Analyzing fecal calprotectin level as a maker of gut inflammation, no statistically significant differences were found between the AD patients and the controls. Actually, the increased fecal calprotectin level $(\geq 50 \mu \mathrm{g} / \mathrm{g})$ was found in 46 vs $60 \%$ of the subjects, receptively $(p=0.275)$. Such a high rate of abnormal results in both groups might be surprising. However, the advanced age of the studied populations has to be taken into consideration. Accordingly to the report by Joshi et al. (2010) the age-related cut-off value of $112 \mu \mathrm{g} / \mathrm{g}$ (instead of $50 \mu \mathrm{g} / \mathrm{g}$ ) for fecal calprotectin should be applied in subjects above 60 years of age. In the present study, the fecal calprotectin level was $<112 \mu \mathrm{g} / \mathrm{g}$ in $74 \%$ of the $\mathrm{AD}$ patients and $80 \%$ of the controls. The fecal calprotectin level exceeded $150 \mu \mathrm{g} / \mathrm{g}$ in $8 \mathrm{AD}$ patients (23\%) and only in two control subject, but this difference did not reach statistical significance. Fecal calprotectin as a marker of the gut immune system activation is composed of S100A8 and S100A9 proteins. Due to the intrinsically amyloidogenic amino acid sequences of these proteins, they can form amyloid oligomers and fibrils closely resembling amyloid polypeptides such as $\alpha$-syn and amyloid $\beta$ (Kowalski and Mulak 2019). Therefore, the intestinal calprotectin pool could contribute to amyloid formation both in the ENS and the CNS.
Indeed, the increased S100A9 level in the cerebrospinal fluid in AD patients was found (Horvath et al. 2016), while in one earlier study the increased level of fecal calprotectin in $\mathrm{AD}$ patients was also reported (Leblhuber et al. 2015). Moreover, elevated fecal calprotectin level has been observed in PD patients too (Schwiertz et al. 2018; Mulak et al. 2019).

Analyzing the relation between fecal calprotectin level and SIBO presence as well as other clinical characteristics of the AD patients no significant associations were found. Although alterations in the gut microbiota composition may be directly connected with the gut immune system activation and disturbed intestinal barrier function (Musa et al. 2019), there are no clear data on the association between SIBO and fecal calprotectin level. Montalto et al. (2008) showed that patients with SIBO are not characterized with a higher fecal calprotectin level, whereas in patients with concomitant Crohn's disease (Ricci et al. 2018) or systemic sclerosis (Marie et al. 2015) SIBO was related to increased fecal calprotectin concentration. In the current study no correlation between fecal calprotectin level and the MMSE score in AD was found that is in line with the earlier report by Leblhuber et al. (2015)

Owing to the fact that all recruited patients were already treated for $\mathrm{AD}$, the influence of $\mathrm{AD}$ medications on gastrointestinal functions and intestinal inflammation needs to be considered while interpreting the results. Indeed, antiinflammatory effect of cholinergic drugs has been shown in animal models of colitis (Pai and Yu 2020). Rivastigmine, an acetylcholinesterase inhibitor, alleviates experimentally induced colitis in rodents by acting at central and peripheral sites to modulate immune responses (Shifrin et al. 2013). Also galantamine induces anti-inflammatory effect in a rat model of colitis involving alpha-7 nicotinic acetylcholine receptor $(\alpha 7 \mathrm{nAChR})$ to suppress pro-inflammatory cytokines (Wazea et al. 2018). In another murine model of colitis, pyridostigmine bromide has been shown to promote mucin synthesis, suppress Th2-dependent inflammation, and attenuate dysbiosis (Singh et al 2020). The above-mentioned effects of the drugs used in the treatment of $\mathrm{AD}$ are directly related to the concept of cholinergic anti-inflammatory pathway that is an efferent vagus nerve-based mechanism which regulates immune responses and cytokine production through $\alpha 7 \mathrm{nAChR}$ signaling (Ji et al. 2014; Bonaz et al. 2016). Interestingly, also memantine acting by blocking N-methyl-Daspartate (NMDA) receptors significantly attenuates macroscopic and microscopic signs of colitis in a mouse model decreasing the plasma levels of interleukin- $1 \beta$, interleukin- 6 , and colon level of tumor necrosis factor- $\alpha$ and myeloperoxidase (Motaghi et al. 2016). A potential influence of pharmacotherapy used for $\mathrm{AD}$ on investigated fecal markers of gut inflammation and intestinal permeability could result in detecting no significant differences between the AD patients and the controls. To elucidate the role of gut inflammation in 
the pathogenesis of $\mathrm{AD}$, these markers should be evaluated in de novo diagnosed patients before initiating the treatment.

Zonulin known as a protein regulating intestinal permeability by the modulation of tight junctions has been used so far as a marker of intestinal integrity and permeability (Fasano 2012). Only recently, one of the commercially available 'zonulin' ELISA assay, also used in the present study (IDK ${ }^{\circledR}$ Zonulin, Immundiagnostik AG, Germany), has been verified to detect complement $\mathrm{C} 3$ instead of the actual protein, prehaptoglobin-2 (Ajamian et al. 2019). A potential role of complement $\mathrm{C} 3$ in the regulation of the gut barrier function remains unclear. It has also been suggested that complement $\mathrm{C} 3$ might be an unspecific product overshadowing the real targets detected by the ELISA assays including one or more members of the zonulin family that have not been discovered yet (Fasano 2020). Due to the methodological limitation of the used zonulin ELISA test, although we decided to present the obtained data, we refrain from the conclusive interpretation.

Apart from methodological limitations related to fecal zonulin evaluation, other constraints of this preliminary study include relatively small number of the recruited subjects, as well as the fact that all AD patients were already treated. Therefore, the influence of AD medications such as cholinergic drugs on gastrointestinal functions and intestinal inflammation constitutes an important confounding factor. Another weakness of the study is the assessment of SIBO itself, since the accuracy of the used LHBT with a reported specificity of $86 \%$ and sensitivity of $52 \%$ remains limited (Tan et al. 2014; Bures et al. 2010; Khoshini et al. 2008; Quigley and Abu-Shanab 2010). Nevertheless, this test is still the most widely used technique. The evaluation of SIBO based on the colony counts in the duodenal aspirates is invasive and has also other drawbacks (Bohm et al. 2013). Despite the above shortcomings, this study presents novel data on SIBO presence in AD patients evaluated concomitantly with fecal calprotectin.

In conclusion, the prevalence of SIBO was significantly higher in the $\mathrm{AD}$ patients than in age-matched controls without dementia (49 vs $22 \%$ ). There was no statistically significant difference in fecal calprotectin level in the AD patients compared to the controls which may result from anti-inflammatory effect of cholinergic drugs used in the treatment of $\mathrm{AD}$. In the $\mathrm{AD}$ patients, there was no association between the presence of SIBO and fecal calprotectin level, nor the MMSE score. Moreover, no correlation between fecal calprotectin level and the MMSE score in AD was found. Further studies including interventional trials in patients with newly diagnosed $\mathrm{AD}$ should help to unravel a causal association between SIBO, gut inflammation and neurodegenerative processes.
Acknowledgements The authors wish to thank Dr Maria Jasińska for her expert technical assistance. We thank the Statistical Analysis Center at Wroclaw Medical University for help in the data analysis. We are also grateful to the staff of the Alzheimer's Disease Research Center at Wroclaw Medical University, Ścinawa, Poland for support in organizing the study.

Author contributions KK: research design, data collection and analysis, writing the manuscript; AM: research conception and design, data evaluation, writing and revising the manuscript. Both authors have read and approved the final version of the manuscript.

Funding This study was supported by a research grant from Wroclaw Medical University (SUB.C131.21.043).

Data availability The datasets generated during the current study are available upon request.

Code availability N/A.

\section{Declarations}

Conflict of interest The authors declare that they have no conflict of interest.

Ethical approval The protocol of this study was approved by the local Ethics Committee (KB-491/2017).

Consent to participate A written informed consent was obtained from all participants prior to the study enrollment.

Consent for publication N/A.

Open Access This article is licensed under a Creative Commons Attribution 4.0 International License, which permits use, sharing, adaptation, distribution and reproduction in any medium or format, as long as you give appropriate credit to the original author(s) and the source, provide a link to the Creative Commons licence, and indicate if changes were made. The images or other third party material in this article are included in the article's Creative Commons licence, unless indicated otherwise in a credit line to the material. If material is not included in the article's Creative Commons licence and your intended use is not permitted by statutory regulation or exceeds the permitted use, you will need to obtain permission directly from the copyright holder. To view a copy of this licence, visit http://creativecommons.org/licenses/by/4.0/.

\section{References}

Ajamian M, Steer D, Rosella G, Gibson PR (2019) Serum zonulin as a marker of intestinal mucosal barrier function: may not be what it seems. PLoS ONE. https://doi.org/10.1371/journal.pone.0210728

Akbari E, Asemi Z, DaneshvarKakhaki R et al (2016) Effect of probiotic supplementation on cognitive function and metabolic status in Alzheimer's disease: a randomized, double-blind and controlled trial. Front Aging Neurosci. https://doi.org/10.3389/fnagi.2016. 00256

Almeida JA, Kim R, Stoita A, McIver CJ, Kurtovic J, Riordan SM (2008) Lactose malabsorption in the elderly: role of small intestinal bacterial overgrowth. Scand J Gastroenterol 43:146-154. https://doi.org/10.1080/00365520701676617 
Armstrong RA (2019) Risk factors for Alzheimer's disease. Folia Neuropathol 57:87-105. https://doi.org/10.5114/fn.2019.85929

Bohm M, Siwiec RM, Wo JM (2013) Diagnosis and management of small intestinal bacterial overgrowth. Nutr Clin Pract 28:289-299. https://doi.org/10.1177/0884533613485882

Bonaz B, Sinniger V, Pellissier S (2016) Anti-inflammatory properties of the vagus nerve: potential therapeutic implications of vagus nerve stimulation. J Physiol 594:5781-5790. https://doi.org/10. 1113/JP271539

Bures J, Cyrany J, Kohoutova D et al (2010) Small intestinal bacterial overgrowth syndrome. World J Gastroenterol 16:2978-2990. https://doi.org/10.3748/wjg.v16.i24.2978

Choung RS, Ruff KC, Malhotra A et al (2011) Clinical predictors of small intestinal bacterial overgrowth by duodenal aspirate culture. Aliment Pharmacol Ther 33:1059-1067. https://doi.org/10.1111/j. 1365-2036.2011.04625.x

Colovic MB, Krstic DZ, Lazarevic-Pasti TD, Bondzic AM, Vasic VM (2013) Acetylcholinesterase inhibitors: pharmacology and toxicology. Curr Neuropharmacol 11:315-335. https://doi.org/10.2174/ 1570159X11311030006

Dinan TG, Cryan JF (2017) Gut instincts: microbiota as a key regulator of brain development, ageing and neurodegeneration. J Physiol 595:489-503. https://doi.org/10.1113/jp273106

Elphick DA, Chew TS, Higham SE et al (2005) Small bowel bacterial overgrowth in symptomatic older people: can it be diagnosed earlier? Gerontology 51:396401. https://doi.org/10.1159/000088704

Fasano A (2012) Zonulin, regulation of tight junctions, and autoimmune diseases. Ann NY Acad Sci 1258:25-33. https://doi.org/10. 1111/j.1749-6632.2012.06538.x

Fasano A (2020) All disease begins in the (leaky) gut: role of zonulinmediated gut permeability in the pathogenesis of some chronic inflammatory diseases. F1000 Res. https://doi.org/10.12688/f1000 research.20510.1

Fasano A, Bove F, Gabrielli M et al (2013) The role of small intestinal bacterial overgrowth in Parkinson's disease. Mov Disord 28:1241-1249. https://doi.org/10.1002/mds.25522

Ferreira-Vieira TH, Guimaraes IM, Silva FR, Ribeiro FM (2016) Alzheimer's disease: targeting the cholinergic system. Curr Neuropharmacol 14:101-105. https://doi.org/10.2174/1570159x1366615 0716165726

Friedland RP (2015) Mechanisms of molecular mimicry involving the microbiota in neurodegeneration. J Alzheimers Dis 45:349-362. https://doi.org/10.3233/jad-142841

Friedland RP, Chapman MR (2017) The role of microbial amyloid in neurodegeneration. PLoS Pathog 13:e1006654. https://doi.org/10. 1371/journal.ppat.1006654

Gabrielli M, Bonazzi P, Scarpellini E et al (2011) Prevalence of small intestinal bacterial overgrowth in Parkinson's disease. Mov Disord 26:889892. https://doi.org/10.1002/mds.23566

Hampel H, Mesulam M-M, Cuello AC et al (2018) The cholinergic system in the pathophysiology and treatment of Alzheimer's disease. Brain 141:1917-1933. https://doi.org/10.1093/brain/awy132

He Y, Li B, Sun D, Chen S (2020) Gut microbiota: implications in Alzheimer's disease. J Clin Med. https://doi.org/10.3390/jcm9072042

Horvath I, Jia X, Johansson P et al (2016) Pro-inflammatory S100A9 protein as a robust biomarker differentiating early stages of cognitive impairment in Alzheimer's disease. ACS Chem Neurosci 7:34-39. https://doi.org/10.1021/acschemneuro.5b00265

Ji H, Rabbi MF, Labis B et al (2014) Central cholinergic activation of a vagus nerve-to-spleen circuit alleviates experimental colitis. Mucosal Immunol 7:335-347. https://doi.org/10.1038/mi.2013.52

Joshi S, Lewis SJ, Creanor S, Ayling RM (2010) Age-related faecal calprotectin, lactoferrin and tumour M2-PK concentrations in healthy volunteers. Ann Clin Biochem 47:259-263. https://doi. org/10.1258/acb.2009.009061
Jouanne M, Rault S, Voisin-Chiret AS (2017) Tau protein aggregation in Alzheimer's disease: AN attractive target for the development of novel therapeutic agents. Eur J Med Chem 139:153-167. https://doi.org/10.1016/j.ejmech.2017.07.070

Khoshini R, Dai SC, Lezcano S, Pimentel M (2008) A systematic review of diagnostic tests for small intestinal bacterial overgrowth. Dig Dis Sci 53:1443-1454. https://doi.org/10.1007/ s10620-007-0065-1

Köhler C, Maes M, Slyepchenko A et al (2016) The gut-brain axis, including the microbiome, leaky gut and bacterial translocation: mechanisms and pathophysiological role in Alzheimer's disease. Curr Pharm Des 22:6152-6166. https://doi.org/10.2174/13816 12822666160907093807

Kowalski K, Mulak A (2019) Brain-gut-microbiota axis in Alzheimer's disease. J Neurogastroenterol Motil 25:48-60. https://doi. org/10.5056/jnm18087

Kowalski K, Jasińska M, Mulak A (2017) Small intestinal bacterial overgrowth in Alzheimer's disease-a pilot study. Int Soc Microbiota J 4:91

Leblhuber F, Geisler S, Steiner K, Fuchs D, Schütz B (2015) Elevated fecal calprotectin in patients with Alzheimer's dementia indicates leaky gut. J Neural Transm 122:1319-1322. https:// doi.org/10.1007/s00702-015-1381-9

Leblhuber F, Strasser B, Steiner K et al (2017) On the role of intestinal microbiota in patients with cognitive decline. J Pharm Pharmacol 5:648-653

Liu S, Gao J, Zhu M, Liu K, Zhang HL (2020) Gut microbiota and dysbiosis in Alzheimer's disease: implications for pathogenesis and treatment. Mol Neurobiol 57:5026-5043. https://doi.org/10. 1007/s12035-020-02073-3

Lukiw WJ (2016) Bacteroides fragilis lipopolysaccharide and inflammatory signaling in Alzheimer's disease. Front Microbiol 7:1544. https://doi.org/10.3389/fmicb.2016.01544

Marie I, Leroi A-M, Menard J-F, Levesque H, Quillard M, Ducrotte $P$ (2015) Fecal calprotectin in systemic sclerosis and review of the literature. Autoimmun Rev 14:547-554. https://doi.org/10. 1016/j.autrev.2015.01.018

Martorana A, Esposito Z, Koch G (2010) Beyond the cholinergic hypothesis: do current drugs work in Alzheimer's disease? CNS Neurosci Ther 16:235-245. https://doi.org/10.1111/j.17555949.2010.00175.x

Mimica N, Presecki P (2009) Side effects of approved antidementives. Psychiatr Danub 21:108-113

Montalto M, Santoro L, Dalvai S et al (2008) Fecal calprotectin concentrations in patients with small intestinal bacterial overgrowth. Dig Dis 26:183-186. https://doi.org/10.1159/00011 6777

Montalto M, Gallo A, Santoro L et al (2013) Role of fecal calprotectin in gastrointestinal disorders. Eur Rev Med Pharmacol Sci 17:1569-1582

Motaghi E, Hajhashemi V, Mahzouni P, Minaiyan M (2016) The effect of memantine on trinitrobenzene sulfonic acid-induced ulcerative colitis in mice. Eur J Pharmacol 793:28-34. https://doi.org/10. 1016/j.ejphar.2016.10.032

Mulak A, Koszewicz M, Panek-Jeziorna M, Koziorowska-Gawron E, Budrewicz S (2019) Fecal calprotectin as a marker of the gut immune system activation is elevated in Parkinson's disease. Front Neurosci. https://doi.org/10.3389/fnins.2019.00992

Musa MA, Kabir M, Hossain MI et al (2019) Measurement of intestinal permeability using lactulose and mannitol with conventional five hours and shortened two hours urine collection by two different methods: HPAE-PAD and LC-MSMS. PLoS ONE 14:e0220397. https://doi.org/10.1371/journal.pone.0220397

Newberry C, Tierney A, Pickett-Blakely O (2016) Lactulose hydrogen breath test result is associated with age and gender. Biomed Res Int. https://doi.org/10.1155/2016/1064029 
Niu XL, Liu L, Song ZX et al (2016) Prevalence of small intestinal bacterial overgrowth in Chinese patients with Parkinson's disease. J Neural Transm 123:1381-1386. https://doi.org/10.1007/ s00702-016-1612-8

Ohlsson B, Roth B, Larsson E, Höglund P (2017) Calprotectin in serum and zonulin in serum and feces are elevated after introduction of a diet with lower carbohydrate content and higher fiber, fat and protein contents. Biomed Rep 6:411-422. https://doi.org/10.3892/ br.2017.865

Oveisgharan S, Arvanitakis Z, Yu L, Farfel J, Schneider JA, Bennett DA (2018) Sex differences in Alzheimer's disease and common neuropathologies of aging. Acta Neuropathol 136:887-900. https://doi.org/10.1007/s00401-018-1920-1

Pai YC, Yu LCH (2020) Is "cholinergic" stimulus useful for ulcerative colitis treatment? Dig Dis Sci 65:6-8. https://doi.org/10.1007/ s10620-019-05933-8

Parlesak A, Klein B, Schecher K, Bode JC, Bode C (2003) Prevalence of small bowel bacterial overgrowth and its association with nutrition intake in nonhospitalized older adults. J Am Geriatr Soc 51:768-773. https://doi.org/10.1046/j.1365-2389.2003.51259.x

Puig KL, Combs CK (2013) Expression and function of APP and its metabolites outside the central nervous system. Exp Gerontol 48:608-611. https://doi.org/10.1016/j.exger.2012.07.009

Quigley EMM (2017) Microbiota-brain-gut axis and neurodegenerative diseases. Curr Neurol Neurosci Rep 17:94. https://doi.org/10. 1007/s11910-017-0802-6

Quigley EMM (2019) The spectrum of small intestinal bacterial overgrowth (SIBO). Curr Gastroenterol Rep 21:3. https://doi.org/10. 1007/s11894-019-0671-z

Quigley EM, Abu-Shanab A (2010) Small intestinal bacterial overgrowth. Infect Dis Clin North A 24:943-959. https://doi.org/10. 1016/j.idc.2010.07.007

Reitz C, Mayeux R (2014) Alzheimer disease: epidemiology, diagnostic criteria, risk factors and biomarkers. Biochem Pharmacol 88:640-651. https://doi.org/10.1016/j.bcp.2013.12.024

Ricci JER, Chebli LA, Ribeiro TCDR et al (2018) Small-intestinal bacterial overgrowth is associated with concurrent intestinal inflammation but not with systemic inflammation in Crohn's disease patients. J Clin Gastroenterol 52:530-536. https://doi.org/10.1097/ mcg.0000000000000803

Schwiertz A, Spiegel J, Dillmann U et al (2018) Fecal markers of intestinal inflammation and intestinal permeability are elevated in Parkinson's disease. Parkinsonism Relat Disord 50:104-107. https://doi.org/10.1016/j.parkreldis.2018.02.022

Semar S, Klotz M, Letiembre M et al (2013) Changes of the enteric nervous system in amyloid- $\beta$ protein precursor transgenic mice correlate with disease progression. J Alzheimers Dis 36:7-20. https://doi.org/10.3233/jad-120511

Shifrin H, Nadler-Milbauer M, Shoham S, Weinstock M (2013) Rivastigmine alleviates experimentally induced colitis in mice and rats by acting at central and peripheral sites to modulate immune responses. PLoS ONE 8(2):e57668. https://doi.org/10.1371/journ al.pone. 0057668

Singh SP, Chand HS, Banerjee S et al (2020) Acetylcholinesterase inhibitor pyridostigmine bromide attenuates gut pathology and bacterial dysbiosis in a murine model of ulcerative colitis. Dig Dis Sci 65:141-149. https://doi.org/10.1007/s10620-019-05838-6

Sochocka M, Donskow-Łysoniewska K, Diniz BS et al (2019) The gut microbiome alterations and inflammation-driven pathogenesis of Alzheimer's disease - a critical review. Mol Neurobiol 56:18411851. https://doi.org/10.1007/s12035-018-1188-4

Tamtaji OR, Heidari-soureshjani R, Mirhosseini N et al (2019) Probiotic and selenium co-supplementation, and the effects on clinical, metabolic and genetic status in Alzheimer's disease: a randomized, double-blind, controlled trial. Clin Nutr 38:2569-2575. https://doi.org/10.1016/j.clnu.2018.11.034

Tan AH, Mahadeva S, Thalha AM et al (2014) Small intestinal bacterial overgrowth in Parkinson's disease. Park Relat Disord 20:535540. https://doi.org/10.1016/j.parkreldis.2014.02.019

Tom SE, Hubbard RA, Crane K et al (2015) Characterization of dementia and Alzheimer's disease in an older population: updated incidence and life expectancy with and without dementia. Am J Public Health 105:408-413. https://doi.org/10.2105/ajph.2014.301935

Wazea SA, Wadie W, Bahgat AK, El-Abhar HS (2018) Galantamine anti-colitic effect: role of alpha-7 nicotinic acetylcholine receptor in modulating Jak/STAT3, NF- $\mathrm{BB} / \mathrm{HMGB} 1 / \mathrm{RAGE}$ and p-AKT/Bcl-2 pathways. Sci Rep 8:5110. https://doi.org/10.1038/ s41598-018-23359-6

Zhan X, Stamova B, Sharp FR (2018) Lipopolysaccharide associates with amyloid plaques, neurons and oligodendrocytes in Alzheimer's disease brain: a review. Front Aging Neurosci 10:1-14. https://doi.org/10.3389/fnagi.2018.00042

Zhao Y, Dua P, Lukiw WJ (2015) Microbial sources of amyloid and relevance to amyloidogenesis and Alzheimer's disease (AD). J Alzheimers Dis Parkinsonism. https://doi.org/10.4172/2161-0460. 1000177

Zhao Y, Jaber V, Lukiw WJ (2017) Secretory products of the human GI tract microbiome and their potential impact on Alzheimer's disease (AD): detection of lipopolysaccharide (LPS) in AD hippocampus. Front Cell Infect Microbiol 7:318. https://doi.org/10. 3389/fcimb.2017.00318

Zhu F, Li C, Chu F, Tian X, Zhu J (2020) Target dysbiosis of gut microbes as a future therapeutic manipulation in Alzheimer's disease. Front Aging Neurosci. https://doi.org/10.3389/fnagi.2020. 544235

Publisher's Note Springer Nature remains neutral with regard to jurisdictional claims in published maps and institutional affiliations. 